\title{
Concentrated pineapple juice for visualisation of the oesophagus during magnetic resonance angiography before atrial fibrillation radiofrequency catheter ablation
}

Riccardo Faletti ${ }^{1}$, Marco Gatti ${ }^{1 *} \mathbb{D}$, Andrea Di Chio ${ }^{1}$, Marco Fronda ${ }^{1}$, Matteo Anselmino ${ }^{2}$, Federico Ferraris ${ }^{2}$, Fiorenzo Gaita ${ }^{2}$ and Paolo Fonio ${ }^{1}$

\begin{abstract}
The purpose of this study was to compare in vitro pineapple juice and a solution of concentrated pineapple juice with a paramagnetic contrast agent in order to determine the feasibility of using the solution of concentrated pineapple juice in vivo for oesophagus visualisation at magnetic resonance angiography (MRA) before the radiofrequency catheter ablation procedure for atrial fibrillation. The pineapple juice was concentrated by a microwave heating evaporation process performed in a domestic microwave oven. Five grams of modified potato starch for every $40 \mathrm{~mL}$ of concentrated pineapple juice were added to the concentrated pineapple juice in order to thicken the solution. The solution resulted visually and quantitatively as hyperintense as the contrast agent in vitro (ratio $=1.02$ ). in vivo, no technical difficulties were encountered during the MRA acquisition and a complete enhanced oesophagus was obtained in 37/38 patients (97.4\%). The volumetric analysis and the three-dimensional reconstruction were feasible; the quality was rated as diagnostic in every patient. The intensified oesophagus was successfully merged into the electro-anatomical maps in all the patients. In summary, we demonstrated that this technique allows a feasible and safe oesophagus visualisation during MRA.
\end{abstract}

Keywords: Atrial fibrillation, Catheter ablation, Contrast media, Magnetic resonance imaging, Oesophagus

\section{Key points}

- The imaging of the oesophagus may contribute to improving the safety of the radiofrequency catheter ablation procedure for atrial fibrillation.

- The concentrated pineapple juice resulted visually and quantitatively as hyperintense as the magnetic resonance contrast agent in vitro.

- The oesophagus was visualised and easily merged into the electro-anatomical maps in all the patients.

- A pre-procedural assessment based on the execution of a MRA with oral concentrated pineapple for visualisation of the oesophagus could represent the all-in-one pre-procedural imaging examination

\footnotetext{
*Correspondence: marcogatti17@gmail.com

${ }^{1}$ Radiology Unit, Department of Surgical Sciences, University of Turin, Via

Genova 3, 10126 Turin, Italy

Full list of author information is available at the end of the article
}

before radiofrequency catheter ablation for atrial fibrillation.

\section{Background}

Radiofrequency catheter ablation (RFCA) of atrial fibrillation (AF) has become a common ablation procedure performed worldwide [1]. Asymptomatic ulceration or haemorrhagic thermal lesions of the oesophagus are seen in up to $15 \%$ of patients following $\mathrm{AF}$ ablation [2], this rarely leading to delayed fistula formation with the left atrium (LA). Although the reported worldwide incidence of atrial-oesophageal fistula as a complication of AF ablation is low $(0.03-0.2 \%)$, it has a high mortality and morbidity rate [3].

Computed tomography angiography and magnetic resonance angiography (MRA) are the standard of care imaging techniques for LA and pulmonary vein visualisation [4-8] and the imaging of the oesophagus 
may contribute to improving the safety of procedures $[4-7,9-11]$.

Several studies considered different natural contrast agents to suppress or enhance the signal of the digestive tube [12-14]. Among them, there are two groups leading to either bright lumen (pineapple, blueberry juice) or dark lumen (tap water, orange juice) on T1-weighted images. Among the ones with a high signal on T1-weighted images, both Espinosa et al. [15] and Arthurs et al. [16] found pineapple to be the fruit that contains the highest concentration of $\mathrm{Mn}$ when compared with other fruits analysed in vitro. In addition, it is well known that during the concentrating process, the water is partially removed in the form of vapour from a boiling solution, while solid compositions such as vitamins, minerals and sugars do not change [17].

The purpose of this study was to evaluate the feasibility of the oesophagus visualisation with oral administration of a solution of concentrated pineapple juice during MRA.

\section{Methods \\ In vitro study \\ Study design}

One pint $(473.716 \mathrm{~mL})$ of pineapple juice placed in a glass container was concentrated by a microwave heating evaporation process performed in a domestic microwave oven (MS11K3000AS, Samsung, Maetan-dong, Yeongtong District, Suwon, South Korea). The microwave's power was set to $850 \mathrm{~W}$ for $25 \mathrm{~min}$. Five grams of modified potato starch (Gel'M instantané ed. végétal, Nutrisens MEDICAL, Francheville, France) for every $40 \mathrm{~mL}$ of concentrated pineapple juice were added to the concentrated pineapple juice in order to thicken the solution (Fig. 1).
To evaluate the reproducibility of the microwave heating evaporation processes, we compared five $10-\mathrm{mL}$ syringes filled with concentrated pineapple juice plus modified potato starch obtained from five consecutive and independent concentration process (Fig. 2).

To determine whether the solution so obtained retained the paramagnetic characteristics required by magnetic resonance imaging (MRI) contrast agent, we compared it to a contrast agent we routinely use for MRA (Fig. 3). We scanned three $10-\mathrm{mL}$ syringes filled with: (1) pineapple juice; (2) concentrated pineapple juice plus modified potato starch; and (3) saline diluted gadoteridol (279.3 mg/ $\mathrm{mL}$, ProHance ${ }^{\circ}$ Bracco Altana Pharma, Constance, Germany) at a $3 \%$ concentration. This concentration was chosen based on the hypothesis that the contrast agent, after being injected at a flow rate of $2.5 \mathrm{~mL} / \mathrm{s}$, is homogeneously mixed with the blood pool on its arrival to the heart; therefore, for a mean cardiac output of $5 \mathrm{~L} / \mathrm{min}$ $(83.3 \mathrm{~mL} / \mathrm{s})$, its concentration is about $2.5 / 83.3=3 \%$.

\section{MRA protocol and image analysis}

MRA was performed with a 1.5-T scanner (Achieva, version 2.6, Philips Medical Systems, Eindhoven, The Netherlands) using a 32-channel body phased-array coil. A three-dimensional (3D) spoiled gradient-echo sequence was acquired in the axial plane, with the following technical parameters: repetition time $3.3 \mathrm{~ms}$; echo time $1.2 \mathrm{~ms}$; flip angle $20^{\circ}$; right to left phase encoding; $512 \times 512$ matrix with an isotropic voxel of $1.5 \mathrm{~mm}^{3}$.

All MRA studies were analysed in consensus by two experienced observers with $>5$ years of experience in cardiovascular MRI using OsiriX MD DICOM Viewer (Pixmeo Inc., Bernex, Switzerland).

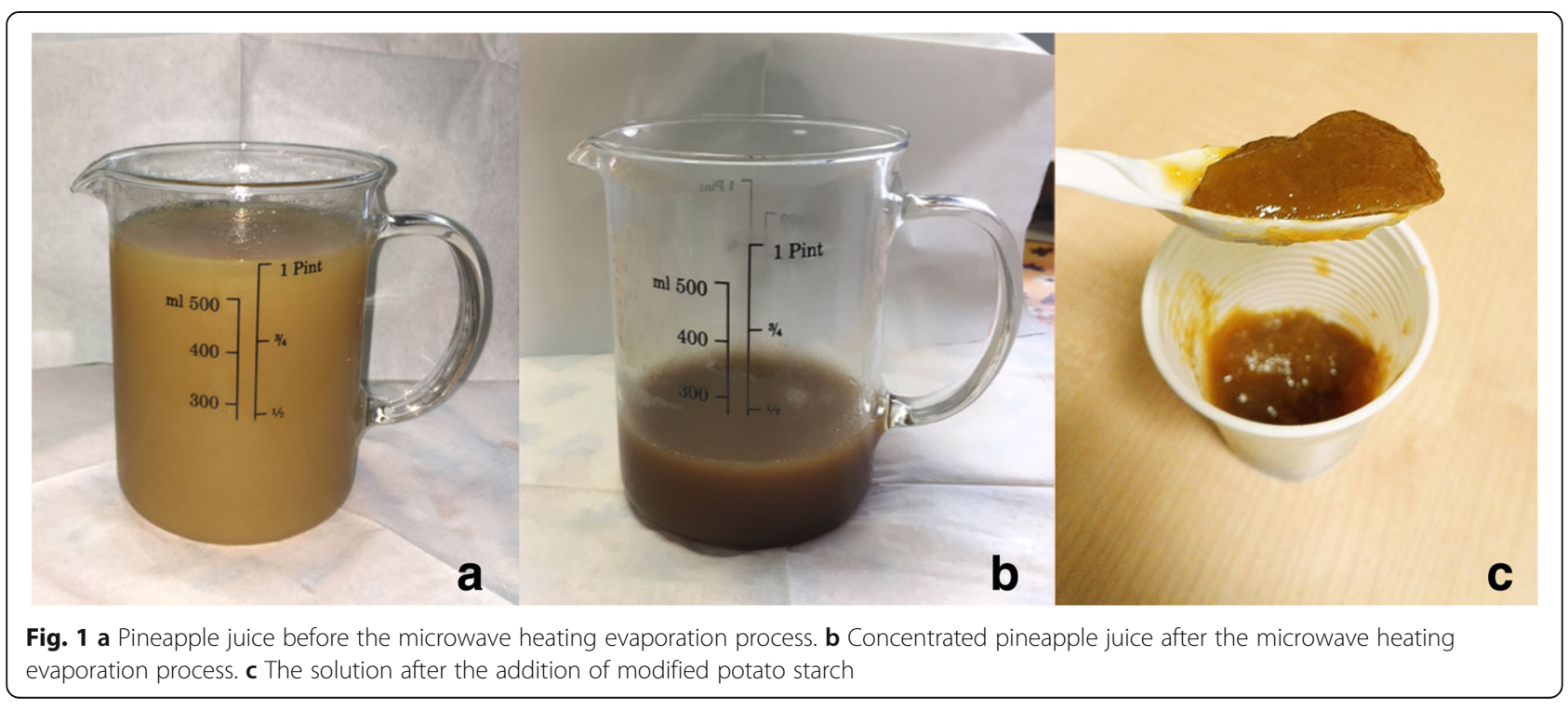




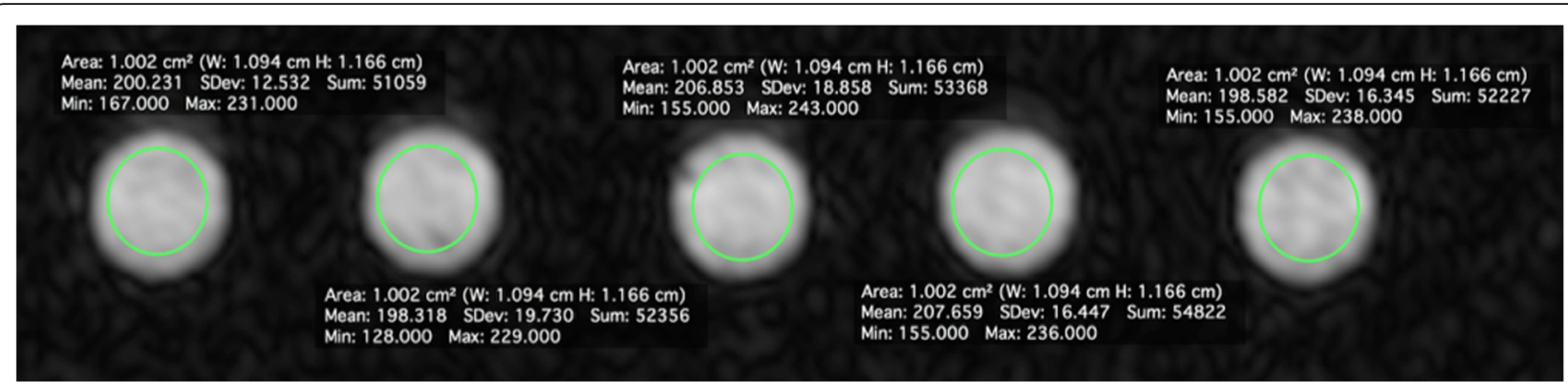

Fig. 2 MRA axial images of the five 10-mL syringe filled concentrated pineapple juice plus modified potato starch

To compare the intensity of the scanned syringe, a region of interest with a mean size of $1 \mathrm{~cm}^{2}$ was placed in the axial plane in the centre of each image (Figs. 2 and 3).

\section{In vivo study}

\section{Study design}

The study was piloted in agreement with the 1964 Helsinki declaration and its later amendments and was approved by the ethics committee of our institution. Before MRA, all patients herein considered were informed about the possible use of their data for study purposes and gave their consent. Patient information was anonymised before the analysis.

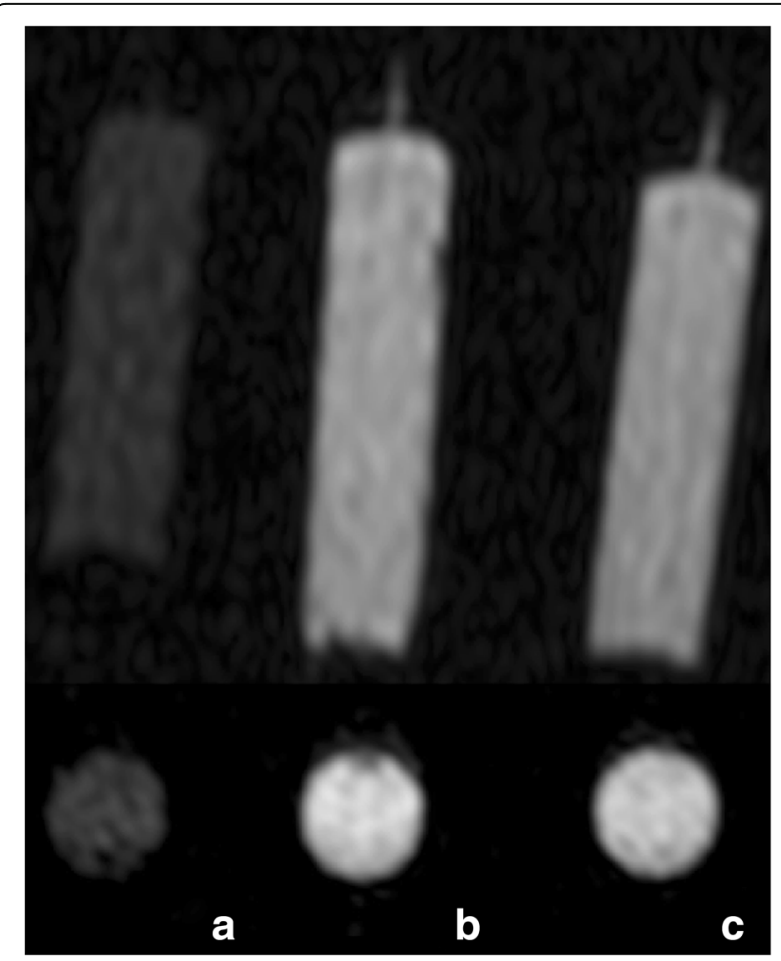

Fig. 3 MRA axial and the corresponding coronal images of three 10-mL syringe filled with: (a) pineapple juice; (b) concentrated pineapple juice plus modified potato starch; and (c) saline diluted gadoteriol at a $3 \%$ concentration
Inclusion criteria were: symptomatic AF refractory to at least one anti-arrhythmic drug; age $\geq 18$ years; and preserved left ventricular ejection fraction at echocardiography. Exclusion criteria were: active hyperthyroidism; impaired left ventricular function; pregnancy; previous oesophageal-gastric surgery; and contraindications to anticoagulation or MRA.

\section{MRA protocol and image analysis}

For the in vivo study, the same not electrocardiographically gated, free-breath sequence was performed after intravenous injection of $0.1 \mathrm{mmol} / \mathrm{kg}$ of gadoteridol at a rate of $2.5 \mathrm{~mL} / \mathrm{s}$, followed by a $20-\mathrm{mL}$ saline bolus at the same rate. The mean sequence time was $25 \mathrm{~s}$ (range 19-32 s). Bolus tracking was used to start the sequence at the exact moment the contrast intensified during the venous phase of pulmonary circulation, to guarantee the maximum signal intensity in the pulmonary veins and into the LA. The oesophagus was intensified by administration of $40 \mathrm{~mL}$ of concentrate pineapple with $5 \mathrm{~g}$ of modified potato starch (Gel'M instantané ed. végétal, Nutrisens MEDICAL, Francheville, France), served with a disposable plastic spoon, while the patients were on the scanning table before the sequence acquisition.

After MRA, all patients were clinically monitored for $30 \mathrm{~min}$. Any adverse effects or anomalies were registered after RFCA before discharge.

For the in vivo study, the feasibility of LA volume and left appendage volume calculation and the quality (classified as diagnostic or non-diagnostic) of 3D maximum intensity projection and $3 \mathrm{D}$ volume rendering reconstructions were evaluated to assess the spatial position of the oesophagus and identify appendage morphology and any anatomical variations of pulmonary veins.

\section{Results}

\section{In vitro study}

Among the five 10-mL syringes filled with concentrated pineapple juice plus modified potato starch, the ratio between the signal intensity measured in the syringe with the lowest signal intensity and the one with the highest 
was $198.138 / 207.659=0.95$ (Fig. 2). The ratio between the signal intensity measured in the syringe filled with pineapple juice and the one with diluted gadoteriol was $59.508 / 198.361=0.30$, while the ratio between the signal intensity of the syringe filled with pineapple juice with the addiction of modified potato starch and the one with diluted gadoteriol was 202.329 / $198.361=1.02$ (Fig. 3).

\section{In vivo study}

Forty patients ( 35 men, mean age 59 years) who met the above-mentioned criteria were enrolled in the study. In all patients, MRA was performed within $24 \mathrm{~h}$ before RFCA. In our series, we had to skip the administration of the oral solution in only two patients, due to known history of severe dysphagia and diabetes. No technical difficulties were encountered during all the MRA acquisitions.

We obtained a complete enhanced oesophagus in 37/38 patients (97.4\%); in the remaining patient, the oesophagus was partially enhanced due to peristaltic waves.

The analysis of LA volume and left appendage volume was feasible in every patient. The quality of $3 \mathrm{D}$ maximum intensity projection and volume rendering reconstruction (Fig. 4), to assess the spatial position of the oesophagus and identify appendage morphology and any pulmonary veins anatomical variations, was rated as diagnostic and the oesophagus was visualised and merged into the electro-anatomical maps generated with Carto $^{\text {Tx }}$ (Biosense Webster, Diamond Bar, CA, USA) in all patients. No immediate or late complication were developed in these patients. In particular, no clinical oesophageal symptoms were observed.

\section{Discussion}

Our study demonstrated that the oral administration of a solution of concentrated pineapple juice for the oesophagus visualisation is a reliable and feasible technique without side effects.

Pineapple juice is widely used as an oral negative contrast agent in MRI cholangiopancreatography [18, 19], sometimes with the addition of gadopentetate dimeglumine $[13,20]$, due to its relatively poor $\mathrm{Mn}$ concentration. On the other side, it is used as an oral positive contrast agent, essentially in the real-time MRI of swallowing $[21,22]$.

The microwave heating evaporation processes herein presented allowed us to obtain, after a few attempts, an appropriate concentrate solution that was as hyperintense as a diluted contrast agent. This is fundamental for obtaining an optimal image quality in vivo, to allow image-reformatting approaches (e.g. 3D reconstruction with variable thickness viewing) and other post-processing methods, as well as the integration of the images into the electro-anatomical maps.

To the best of our knowledge, this is the first experience with the use of a 'natural' concentrated MRI contrast agent. Our group [7] first demonstrated the feasibility of the oesophagus visualisation with oral gadobenate dimeglumine during MRA. In that study, the oesophagus was enhanced using an oral gel solution of $0.7-\mathrm{mL}$ gadobenate dimeglumine contrast agent mixed with $40 \mathrm{mg}$ thickened water gel, which was swallowed by the patients on the scanning table, immediately before the MRA sequence acquisition. With our protocol, we now obtained the same results without the gadobenate dimeglumine.

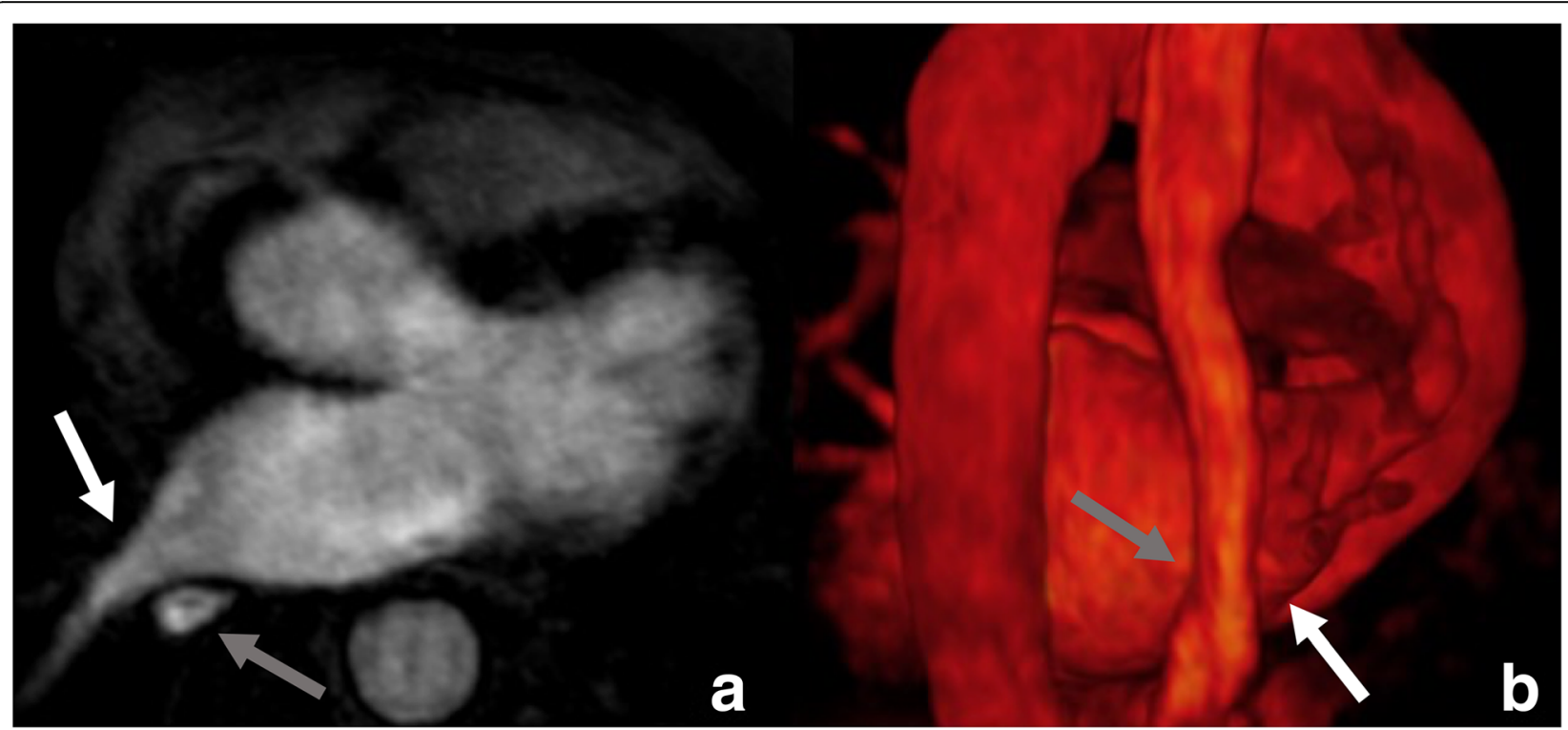

Fig. 4 Axial images (a) with the corresponding 3D volume rendering (b) of the MRA. Note how the oesophagus (grey arrow) courses near the ostia of the right inferior pulmonary vein (white arrow) 
In Europe, the use of macrocyclic agents (gadobutrol, gadoteric acid, and gadoteridol) can continue to be used in their current indications, but in the lowest doses that enhance images sufficiently and only when unenhanced body scans are not suitable [23]. Even if the orally administered Gd-based contrast agent was excreted in almost all the faeces and not absorbed [24], the use of concentrated pineapple juice seems to be a good way of reducing administered dose of contrast media.

Starek et al. [25] reported in their paper that failed oesophagus visualisation was most often related to delayed swallowing of the contrast agent or rapid passage through the oesophagus; therefore, as previously done by our group [7], we first had used thickened gel water, commonly used safely in patients suffering from a swallowing dysfunction and airway problems, and then we used modified potato starch to thicken the concentrated pineapple and with this method we prolonged the oesophageal transit time and allowed the patient to swallow it a few seconds before the administration of contrast agent.

Some studies showed a relatively stable position of the oesophagus in the long term $[6,26]$. Conversely, other studies $[27,28]$ found a poor correlation between the pre-procedural view of the oesophagus and its actual position during the ablation procedure. Recently it has been proven that there are no significant movement of the oesophagus in the short term [25]. Based on this consideration, on the fact that CTA and MRA provide similar information before RFCA [29] and that the contrast-enhanced sequences seem to be highly sensitive and specific for diagnosing LA or LA appendage thrombus [30], a pre-procedural assessment based on an MRA with oral administration of concentrated pineapple for visualisation of the oesophagus a few hours before RFCA could represent the all-in-one pre-procedural imaging examination.

This study has some limitations. First, it is a single-centre study which covers a limited number of patients. Second, we did not directly compare this technique to the similar one based on the administration of a gel solution of gadobenate dimeglumine [7]. Third, we did not do a quantitative analysis in vivo; however, the fact that all the MRA were considered as diagnostic and we were able to merge the imaging into the electro-anatomical maps in all patients, makes us confident of the excellent quality of the in vivo images.

In conclusion, the pineapple juice after an appropriate concentration process and the addiction of modified potato starch is as hyperintense as the MRI diluted contrast media and allows a feasible and safe oesophagus visualisation during MRA.

\section{Abbreviations}

3D: Three-dimensional; AF: Atrial fibrillation; LA: Left atrium; MRA: Magnetic resonance angiography; MRl: Magnetic resonance imaging; RFCA: Radiofrequency catheter ablation

\section{Availability of data and materials}

All data are available on request at the Department of Surgical Sciences, Radiology Unit, "Città della Salute e della Scienza" Hospital, University of Turin, Via Genova 3, 10126, Turin, Italy.

Funding

The authors state that this work has not received any funding.

\section{Acknowledgements}

We acknowledge the collaboration and support of Petrone D and Zamorani D.

\section{Authors' contributions}

RF contributed to the conceptualisation and design of the study and supervised the manuscript editing. MG wrote the first draft of manuscript. AD conceived the idea. $A D, M F$ and $M G$ contributed to the image processing and data analysis. MA and FF contributed to the recruitment of participants and clinical assessments. FG and PF are the guarantors of the study. All authors worked together to develop the experimental design, edited the manuscript and assisted with interpretation of the results. All authors have contributed to and approved the final manuscript.

\section{Ethics approval and consent to participate}

The study was piloted in agreement with the 1964 Helsinki declaration and its later amendments and approved by the local Ethics Committee ( $n^{\circ} 873 / 16$ at Azienda ospedaliero-universitaria Città della Salute e della Scienza di Torino C.so Bramante 88, 10126, Turin, Italy).

\section{Consent for publication}

Written informed consent to participate and to publish results was obtained from all participants.

\section{Competing interests}

The authors declare that they have no competing interests.

\section{Publisher's Note}

Springer Nature remains neutral with regard to jurisdictional claims in published maps and institutional affiliations.

\section{Author details}

'Radiology Unit, Department of Surgical Sciences, University of Turin, Via Genova 3, 10126 Turin, Italy. ${ }^{2}$ Division of Cardiology, Department of Medical Sciences, "Città della Salute e della Scienza" Hospital, University of Turin, Turin, Italy.

Received: 13 July 2018 Accepted: 9 October 2018

Published online: 21 November 2018

\section{References}

1. Calkins H, Kuck KH, Cappato R et al (2012) 2012 HRS/EHRA/ECAS expert consensus statement on catheter and surgical ablation of atrial fibrillation: recommendations for patient selection, procedural techniques, patient management and follow-up, definitions, endpoints, and research trial design. J Interv Card Electrophysiol 33:171-257

2. Halm U, Gaspar T, Zachäus M et al (2010) Thermal esophageal lesions after radiofrequency catheter ablation of left atrial arrhythmias. Am J Gastroenterol 105:551-556

3. Cappato R, Calkins H, Chen SA et al (2010) Updated worldwide survey on the methods, efficacy, and safety of catheter ablation for human atrial fibrillation. Circ Arrhythm Electrophysiol 3:32-38

4. Pollak SJ, Monir G, Chernoby MS, Elenberger CD (2005) Novel imaging techniques of the esophagus enhancing safety of left atrial ablation. J Cardiovasc Electrophysiol 16:244-248

5. Ren JF, Lin D, Marchlinski FE, Callans DJ, Patel V (2006) Esophageal imaging and strategies for avoiding injury during left atrial ablation for atrial fibrillation. Heart Rhythm 3:1156-1161

6. Piorkowski C, Hindricks G, Schreiber D et al (2006) Electroanatomic reconstruction of the left atrium, pulmonary veins, and esophagus compared with the «true anatomy» on multislice computed tomography in patients undergoing catheter ablation of atrial fibrillation. Heart Rhythm 3: 317-327 
7. Faletti R, Rapellino A, Barisone F et al (2014) Use of oral gadobenate dimeglumine to visualise the oesophagus during magnetic resonance angiography in patients with atrial fibrillation prior to catheter ablation. J Cardiovasc Magn Reson 16:41

8. Ohana M, Bakouboula B, Labani A et al (2015) Imaging before and after catheter ablation of atrial fibrillation. Diagn Inter Imaging 96:1113-1123

9. Kenigsberg DN, Lee BP, Grizzard JD, Ellenbogen KA, Wood MA (2007) Accuracy of intracardiac echocardiography for assessing the esophageal course along the posterior left atrium: a comparison to magnetic resonance imaging. J Cardiovasc Electrophysiol 18:169-173

10. Scazzuso FA, Rivera SH, Albina G et al (2013) Three-dimensional esophagus reconstruction and monitoring during ablation of atrial fibrillation: combination of two imaging techniques. Int J Cardiol 168:2364-2368

11. Knecht S, Sticherling C, Reichlin T et al (2017) Reliability of luminal oesophageal temperature monitoring during radiofrequency ablation of atrial fibrillation: insights from probe visualization and oesophageal reconstruction using magnetic resonance imaging. Europace 19:1123-1131

12. Sanchez TA, Elias J Jr, Colnago LA et al (2009) Clinical feasibility of Açai (Euterpe olerácea) pulp as an oral contrast agent for magnetic resonance cholangiopancreatography. J Comput Assist Tomogr 33:666-671

13. Duarte JA, Furtado AP, Marroni CA (2012) Use of pineapple juice with gadopentetate dimeglumine as a negative oral contrast for magnetic resonance cholangiopancreatography: a multicentric study. Abdom Imaging 37:447-456

14. Zhang S, Joseph AA, Gross L, Ghadimi M, Frahm J, Beham AW (2015) Diagnosis of gastroesophageal reflux disease using real-time magnetic resonance imaging. Sci Rep 5:12112

15. Espinosa MG, Sosa M, De León-Rodríguez LM et al (2006) Blackberry (Rubus spp.): a pH-dependent oral contrast medium for gastrointestinal tract images by magnetic resonance imaging. Magn Reson Imaging 24:195-200

16. Arthurs OJ, Graves MJ, Edwards AD, Joubert I, Set PA, Lomas DJ (2014) Interactive neonatal gastrointestinal magnetic resonance imaging using fruit juice as an oral contrast media. BMC Med Imaging 14:33

17. Assawarachan R, Noomhorm A (2010) Changes in color and rheological behavior of pineapple concentrate through various evaporation methods. Int J Agric Biol Eng 3:74-84

18. Bittman ME, Callahan MJ (2014) The effective use of acai juice, blueberry juice and pineapple juice as negative contrast agents for magnetic resonance cholangiopancreatography in children. Pediatr Radiol 44:883-887

19. Riordan RD, Khonsari M, Jeffries J, Maskell GF, Cook PG (2004) Pineapple juice as a negative oral contrast agent in magnetic resonance cholangiopancreatography: a preliminary evaluation. Br J Radiol 77:991-999

20. Coppens E, Metens T, Winant C, Matos C (2005) Pineapple juice labeled with gadolinium: a convenient oral contrast for magnetic resonance cholangiopancreatography. Eur Radiol 15:2122-2129

21. Olthoff A, Joseph AA, Weidenmüller M, Riley B, Frahm J (2016) Real-time MRI of swallowing: intraoral pressure reduction supports larynx elevation. NMR Biomed 29:1618-1623

22. Zhang S, Olthoff A, Frahm J (2012) Real-time magnetic resonance imaging of normal swallowing. J Magn Reson Imaging 35:1372-1379

23. EMA's Pharmacovigilance Risk Assessment Committee (2017) PRAC confirms restrictions on the use of linear gadolinium agents. http://www.ema.europa. eu/docs/en_GB/document_library/Referrals_document/gadolinium_ contrast_agents_31/Recommendation_provided_by_Pharmacovigilance_ Risk_Assessment_Committee/WC500230928.pdf. Accessed 19 May 2018

24. Weinmann HJ, Brasch RC, Press WR, Wesbey GE (1984) Characteristics of gadolinium-DTPA complex: a potential NMR contrast agent. AJR Am J Roentgenol 142:619-624

25. Starek Z, Lehar F, Jez J et al (2017) Three-dimensional rotational angiography of the left atrium and the oesophagus: the short-term mobility of the oesophagus and the stability of the fused three-dimensional model of the left atrium and the oesophagus during catheter ablation for atrial fibrillation. Europace 19:1310-1316

26. Kennedy R, Good E, Oral H et al (2008) Temporal stability of the location of the esophagus in patients undergoing a repeat left atrial ablation procedure for atrial fibrillation or flutter. J Cardiovasc Electrophysiol 19:351-355

27. Daoud EG, Hummel JD, Houmsse M et al (2008) Comparison of computed tomography imaging with intraprocedural contrast esophagram: implications for catheter ablation of atrial fibrillation. Heart Rhythm 5:975-980

28. Nölker G, Gutleben KJ, Marschang H et al (2008) Three-dimensional left atrial and esophagus reconstruction using cardiac C-arm computed tomography with image integration into fluoroscopic views for ablation of atrial fibrillation: accuracy of a novel modality in comparison with multislice computed tomography. Heart Rhythm 5:1651-1657

29. Pontone $G$, Andreini D, Bertella E et al (2015) Comparison of cardiac computed tomography versus cardiac magnetic resonance for characterization of left atrium anatomy before radiofrequency catheter ablation of atrial fibrillation. Int J Cardiol 179:114-121

30. Rathi VK, Reddy ST, Anreddy S et al (2013) Contrast-enhanced CMR is equally effective as TEE in the evaluation of left atrial appendage thrombus in patients with atrial fibrillation undergoing pulmonary vein isolation procedure. Heart Rhythm 10:1021-1027

\section{Submit your manuscript to a SpringerOpen ${ }^{\circ}$ journal and benefit from:}

- Convenient online submission

- Rigorous peer review

- Open access: articles freely available online

High visibility within the field

- Retaining the copyright to your article

Submit your next manuscript at $>$ springeropen.com 